\title{
Numerical modeling of sensors based on long period gratings in photonic crystal fibres
}

Jovana S. Petrovic, Helen Dobb, Vladimir Mezentsev, Kyriacos Kalli, David J. Webb, et al.

Jovana S. Petrovic, Helen Dobb, Vladimir Mezentsev, Kyriacos Kalli, David $\mathrm{J}$. Webb, lan Bennion, "Numerical modeling of sensors based on long period gratings in photonic crystal fibres," Proc. SPIE 6588, Photonic Crystal Fibers, 65880F (4 May 2007); doi: 10.1117/12.722598

SPIE Event: International Congress on Optics and Optoelectronics, 2007, Prague, Czech Republic 


\title{
Numerical modeling of sensors based on long period gratings in photonic crystal fibres
}

\author{
Jovana S. Petrovic*a, Helen Dobb ${ }^{\mathrm{a}}$, Vladimir Mezentseva ${ }^{\mathrm{a}}$ Kyriacos Kallib, David J. Webb ${ }^{\mathrm{a}}$, \\ and Ian Bennion ${ }^{\mathrm{a}}$ \\ ${ }^{a}$ Photonics Research Group, Aston University, Aston Street, B4 7ET Birmingham, UK \\ ${ }^{b}$ Nanophotonics Research Laboratory, Higher Technical Institute, C. Kavafi Str., Aglantzia, P.O. \\ Box 20423, 2152 Nicosia, Cyprus
}

\begin{abstract}
A numerical model of the sensitivity of long period gratings fabricated by electric-arc in photonic crystal fibres to strain, temperature and refractive index is proposed and evaluated by comparison to the experimental results. It is shown to be superior to the commonly used semi-analytical method. The generalized modelling procedure is thoroughly explained in order to facilitate its application to a wide range of long period gratings in different types of fibres.
\end{abstract}

Keywords: photonic crystal fibre, long period grating, sensing

\section{INTRODUCTION}

Long period gratings (LPGs) were first proposed as a solution to high back-reflection in band-rejection filters [1,2]. An LPG consists of a periodic change of the refractive index along the fibre which causes coupling between the core and cladding modes and therefore attenuation bands in the transmission spectrum. As these attenuation bands are sensitive to changes in external parameters such as temperature and refractive index as well as to strain and bending of the fibre, the LPGs have been largely exploited as sensing devices. LPGs have been produced by various methods: UV, $\mathrm{CO}_{2}$ or femtosecond laser writing, exposure to electric arc, mechanical pressure, etching, [3], in different types of fibres: single mode, progressive layered, W-shaped, grapefruit fibres, see e.g. [3-5]. The development of photonic crystal fibres (PCFs), [6], enabled fabrication of a new class of long period gratings. Due to the novel waveguiding properties and the material composition of PCFs, LPGs inscribed in them can be an elegant solution to the problems previously encountered with other LPGs, for example gratings fabricated by an electric arc are largely immune to the crosssensitivity to temperature, [7] and also have excellent thermal stability [8].

Fibre gratings have been successfully modelled by several methods, the most common of which are coupledmode theory (CMT) and the transfer matrix method (TMM) [9,10]. Grating sensitivity to an external parameter has been calculated based on the derivative of its resonant wavelength with respect to that parameter. Since the CMT is a perturbation theory, it can be applied to any fibre providing that its eigenmodes and the index change are known. Due to the complex geometry of PCFs, this is exactly the major challenge. While core modes of PCFs have been extensively analysed by various methods [11-17], cladding modes have been studied only in a few particular contexts mostly related to LPGs $[5,18]$. As the coupling to these modes is a working principle of LPG, accurate calculation of their propagation constants and fields is essential.

In this paper, resonant cladding modes of an LPG fabricated by an electric arc in all-silica PCFs are identified and used in the analysis of the grating sensitivity to temperature, strain and external refractive index. In particular, a very weak grating sensitivity to temperature is proved. The paper begins with a brief review of the relevant experimental results in Section 2. Section 3 describes the numerical model used divided into the model of long period grating, model of PCF and semi-analytical and numerical models of the grating sensitivity. Its applications to the sensitivity to strain, temperature and refractive index are described in detail in Sections 4-6, respectively. Results of the modelling and their comparison to the experimental results are discussed. At the end, Section 7 summarizes conclusions and points out directions for future work.

* petrovij@aston.ac.uk; phone 44121204 3519; fax 441212043682

Photonic Crystal Fibers, edited by Kyriacos Kalli, Proc. of SPIE Vol. 6588,

65880F, (2007) - 0277-786X/07/\$18 - doi: 10.1117/12.722598 


\section{LPG IN ESM-1550-01 PCF}

LPGs in endlessly single mode ESM 1550-01 PCF, first reported in [7], were studied as an excellent example of the grating insensitive to temperature and simultaneously sensitive to other parameters. The fibre was made from pure silica with four rings of hexagonally arranged air holes with the space between adjacent holes being $8 \mu \mathrm{m}$. The central hole was omitted to form the core, Fig. 1a). The filling factor was 0.46 . LPGs with different periods were inscribed by exposing the PCF to the electrical discharge provided by a commercial splicer (Fitel S175 v2000) set to the commercially defined power level 87 and the arc duration of 750ms, [19]. A detailed inspection of the grating by the phase microscope did not reveal any change in the hole size. The LPG with period $500 \mu \mathrm{m}$ and length $25.5 \mathrm{~mm}$ was studied in detail. Its transmission spectrum, shown in Fig. 1b), features two pronounced attenuation bands at $1403 \mathrm{~nm}$ and $1239 \mathrm{~nm}$. Increasing the grating period caused a blue shift of the wavelength spectrum, in contrast to LPG behaviour in conventional single mode fibre. Neither the grating spectrum nor the sensitivity tests indicated any birefringence, therefore the original hexagonal symmetry of the fibre is considered unaffected by the electric arc.

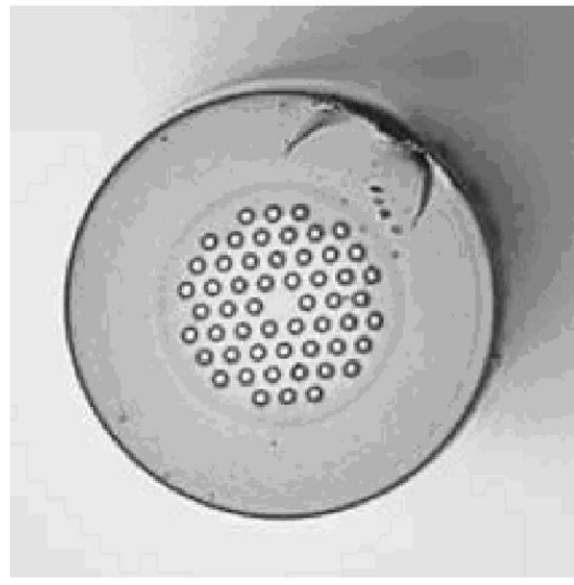

a)

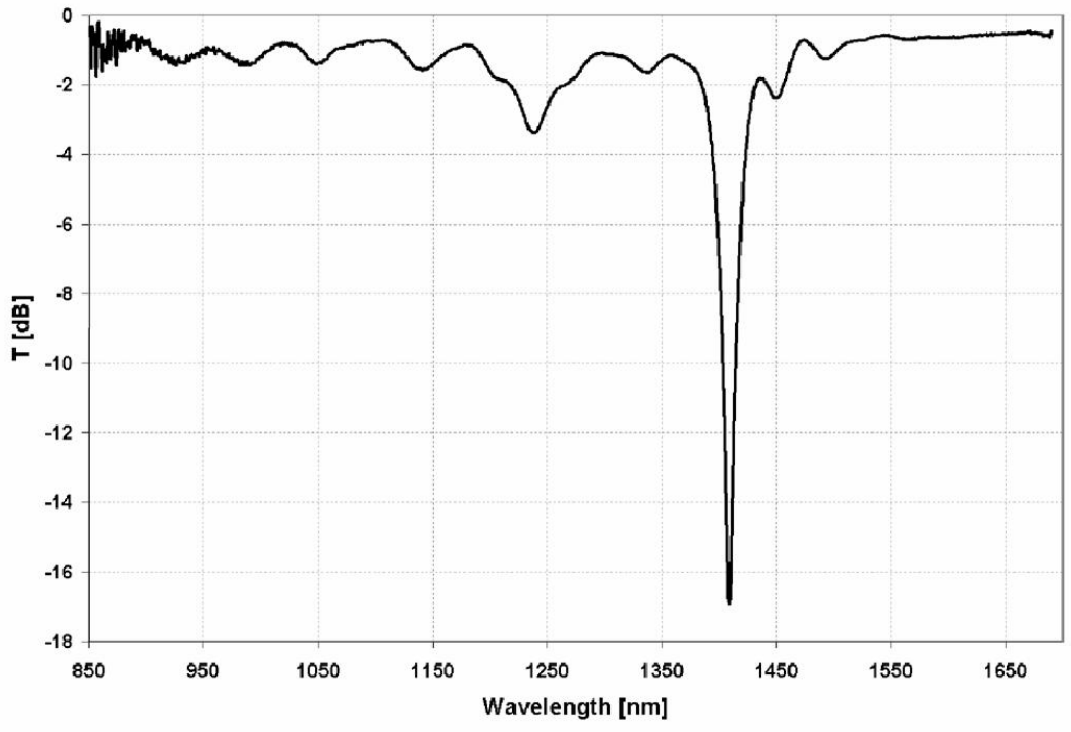

b)

Figure 1. a) Cross section of ESM 1550-01 PCF. b) Spectrum of 500 $\mu \mathrm{m}$ LPG fabricated in this fibre by electric arc

\section{NUMERICAL MODEL}

\subsection{Numerical model of PCF}

Due to the complex geometry of PCFs and the high refractive index contrast between the pure silica and air holes, modes of these fibres are best found by solving Maxwell's equations by a full-vectorial numerical solver. A comparison of the different numerical methods used to study PCFs can be found in [16]. In order to simulate an LPG and its sensitivity to refractive index, cladding modes of the stripped fibre exposed to different surrounding media must be found, which automatically eliminates methods that assume infinite photonic crystal. An accurate calculation of the effective refractive indices of the modes propagating mostly through the photonic crystal requires very fine meshing in this part of the fibre cross section. In order to fulfill both requirements, the finite element method was chosen and the simulations were performed by the commercial software Comsol 3.1 [20]. Necessary refinement of the mesh was achieved by exploiting the fibre symmetry. 
The photonic crystal fibre studied in this paper and shown in Fig. 1a) belongs to the $C_{6 v}$ symmetry group. Modes of a waveguide with this symmetry can be divided into eight symmetry classes: non-degenerate classes $1,2,7,8$ with the irreducible zone angle of $\pi / 6$ and degenerate classes $3,4,5,6$ with the irreducible zone angle of $\pi / 2$. Four classes in each group are the result of permutations of the two boundary conditions: perfect magnetic conductor $-\vec{n} \times \vec{H}=0$ and perfect electric conductor $-\vec{n} \times \vec{E}=0$, [21]. Immense improvement of the mesh by the use of fibre symmetry is illustrated in Fig. 2. Basic criteria for the choice of mesh parameters were convergence and the required accuracy of the effective refractive index. The optimal mesh is chosen as a compromise between the numerical accuracy and the calculation time. The effective index of the core mode was calculated with the accuracy better than $2 \cdot 10^{-7}$ and of cladding modes with the accuracy better than $10^{-6}$.
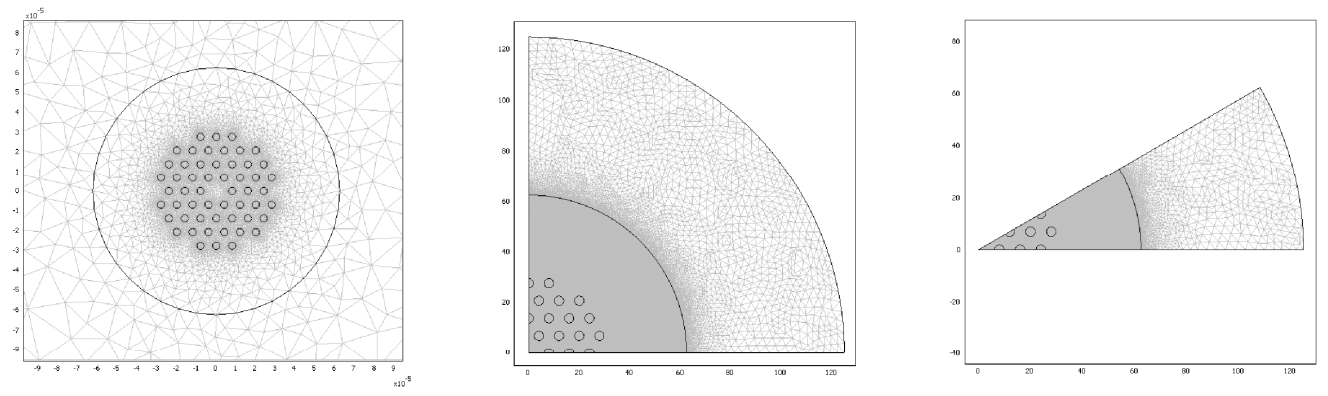

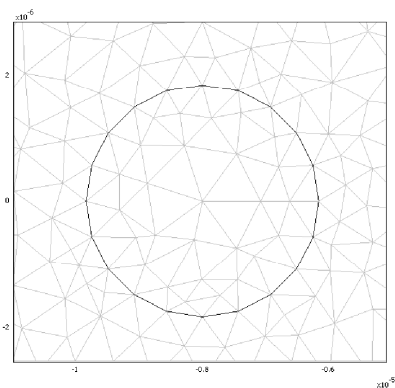

a)

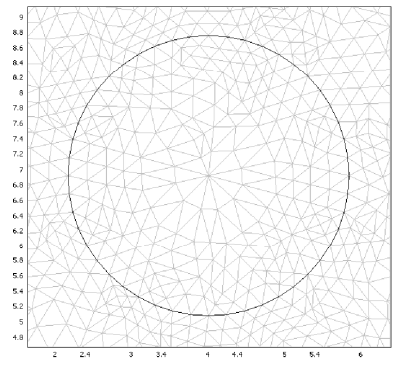

b)

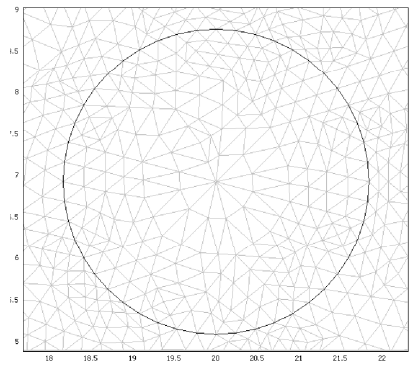

c)

Figure 2. Optimal mesh that could be solved for on the Pentium 4 (512MB RAM, 2.53GHz) in the case of a) the whole fibre cross section and b) degenerate and c) non-degenerate classes. The number of mesh elements in the whole cross section was increased from 19960 to 376048 for degenerate and to 376464 for non-degenerate classes.

\subsection{Grating theory}

If the grating can be treated as a periodic perturbation of the refractive index along the fibre, then a mode excited by the grating can be represented in the space of the eigenmodes of the unperturbed fibre, and the coupling mode theory is an excellent tool for the grating analysis. Long period grating couples core mode to the copropagating cladding modes which are attenuated in the coated part of the fibre. The resonant wavelength at which the coupling occurs is given by the phase matching condition

$$
\lambda=\left(n_{c o}^{e f f}-n_{c l}^{e f f}\right) \Lambda_{B}=\delta n^{e f f} \Lambda_{B}
$$

where $n_{c o}^{e f f}$ and $n_{c l}^{\text {eff }}$ are the effective mode indices of the core and cladding modes respectively, $\Lambda$ is the beat length and $\lambda$ is the resonant wavelength [22]. However, the difference in the effective refractive indices of the successive cladding modes can be of the order of $10^{-6}$ which is not only close to the numerical error, but also does not allow for the separation of contributions of different modes to the same attenuation band. Namely, the attenuation bands of these modes would be separated by a few nanometers, while the width of the spectral band is several nanometers. The mode with the biggest contribution is the mode with the biggest coupling to the core mode, which is quantified by the coupling 
coefficient $\kappa=\frac{\pi \varepsilon_{0} c n}{2 \lambda} \int_{\mathbf{r}} \Delta n(\mathbf{r}) \mathbf{E}_{c o}(\mathbf{r}) \mathbf{E}_{c l}^{*}(\mathbf{r}) d \mathbf{r}$, where $\Delta n$ is amplitude of the index modulation. Indeed, usually the contribution of one mode is so dominant that it can be considered the only resonant mode. As overlap integrals depend on the orientation of mode field vectors, analysis of fibre symmetry can shorten identification of the resonant mode. Numerical error in the calculation of effective indices translates into the inaccuracy in the position of the resonant wavelength of $0.5 \mathrm{~nm}$.

Grating analysis can be facilitated by construction of the grating characteristics which is a family of curves $\Lambda(\lambda)$ for different cladding modes over a range of wavelengths, as shown in Fig. 3 for the ESM 1550-01 PCF. Resonant wavelengths of the first order grating are found at intersections of these curves with the line $\Lambda=L$, where $\mathrm{L}$ is the grating period. Inset in Fig. 3 shows the power profile and the electric field of the quasi LP mode that has been identified as responsible for the main resonance at $1402 \mathrm{~nm}$. The electric field of this mode is parallel to the electric field of the core mode which maximizes their coupling coefficient. The resonant mode has been further used in the models of grating sensitivity to strain, temperature and refractive index.

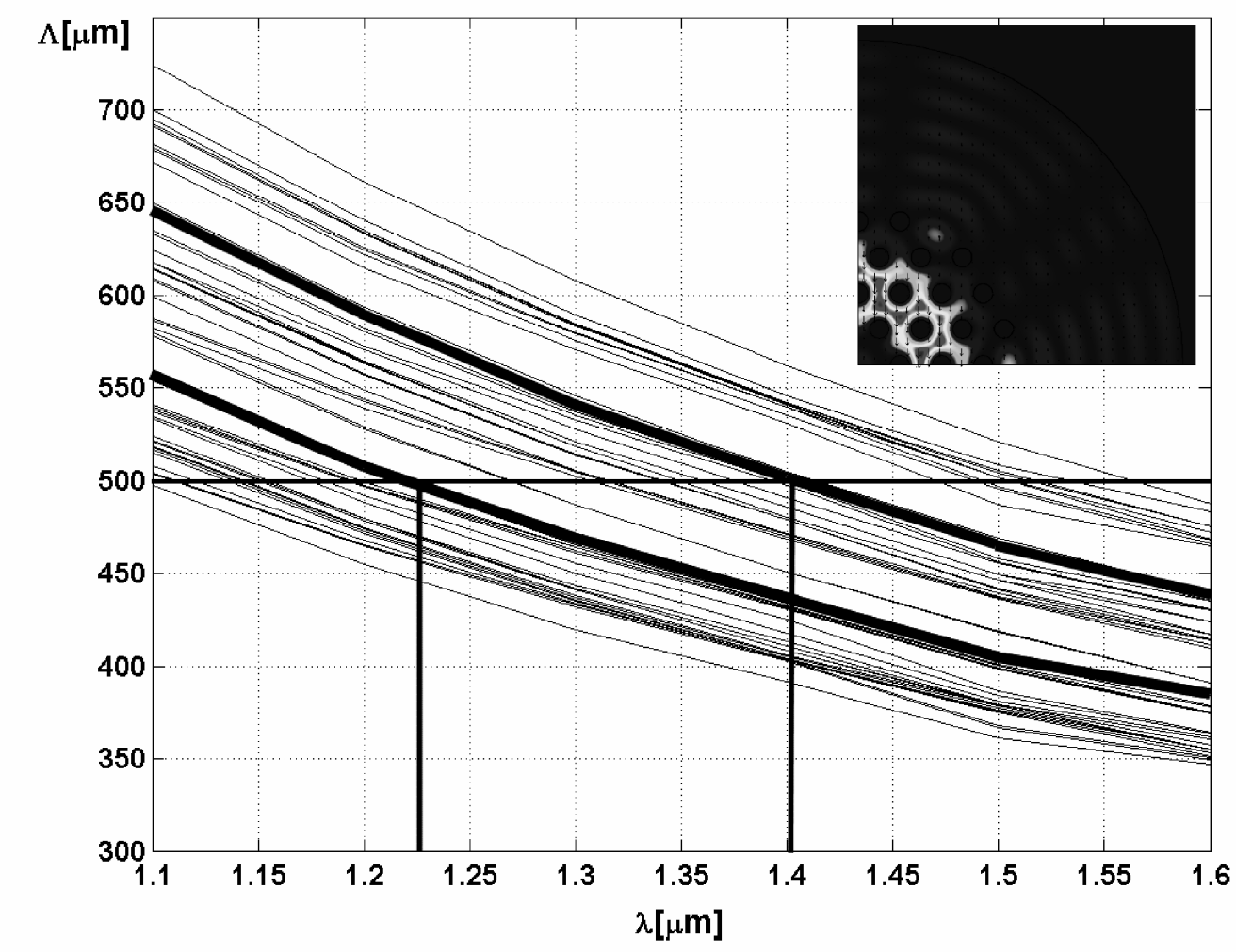

Figure 3. Characteristic of the LPG in ESM 1550-01 PCF. The resonances marked by the vertical lines correspond to the attenuation bands at $1239 \mathrm{~nm}$ and $1403 \mathrm{~nm}$ shown in Fig. 1. Characteristic curves of the resonant modes are emphasized by thick lines. Inset: Power profile and the electric field of the mode resonant at $1403 \mathrm{~nm}$. 


\subsection{Models of grating sensitivity}

Long period grating response to the change in external parameter is observed as a shift in the resonant wavelength, a change in magnitude or in the shape of attenuation bands. In most cases, changes in temperature, strain and refractive index lower than the index of fibre cladding cause wavelength shift, while bending and changes in refractive index to the values higher than the index of the fibre cladding cause a change in shape and magnitude of attenuation bands. In the former case, the grating sensitivity to parameter X can be calculated by linearisation of the grating characteristic of the resonant mode around the resonant wavelength and is given by expression

$$
\frac{\partial \lambda}{\partial X}=\gamma\left(\frac{\partial \Lambda}{\partial X} \delta n^{e f f}+\frac{\partial \delta n^{e f f}}{\partial n} \frac{\partial n}{\partial X} \Lambda\right)
$$

in which the generalized sensing parameter $\gamma[23,24]$

$$
\gamma=\frac{1}{1-\frac{d \delta n^{e f f}}{d \lambda} \Lambda}=\frac{1}{\delta n^{e f f}} \frac{d \lambda}{d \Lambda}
$$

accounts for the influence of the material and waveguide dispersion of the fibre, $\delta n^{\text {eff }}=\delta n^{\text {eff }}(\lambda(X), n(\lambda(X), X))$. The rightmost term in (3) shows a direct relation between $\gamma$ and the slope of the grating characteristics. As the derivatives in the above equations are obtained numerically, this method will be referred to as semi-analytical.

Change in an external parameter can have two major effects on a LPG: change in the grating length and in the refractive index of the fibre. If the latter effect is incorporated in the model of the PCF and the resonant wavelength found using the new grating period, the approximation made in the linear model is eliminated. Graphic representation of this solution clearly shows contributions from each effect as will be shown in Sections 4 and 5. This method will be referred to as numerical.

The most important assumptions made in the model are that the index change in the grating is small and that the symmetry of the fibre is preserved. The profile of the grating and the fibre itself are not restricted in any other way. Moreover, the requirement imposed by symmetry can be dropped at the expense of the mesh quality and the calculation time. Nonuniform grating can be treated by transfer matrix method that uses CMT to evaluate transmission matrix of each uniform part. Therefore, the numerical model proposed here allows for calculation of the wavelength shift based sensitivity of practically any grating in any fibre.

\section{LPG SENSITIVITY TO STRAIN}

The expression for the sensitivity to strain was derived from (2) by substitution of $\mathrm{X}$ by the definition of the linear strain $\varepsilon \equiv \delta \varepsilon=\Delta \Lambda / \Lambda=\Delta L / L$ and is given by

$$
\frac{\partial \lambda}{\partial \varepsilon}=\gamma \lambda\left(1+\frac{\partial \delta n^{e f f}}{\partial n} \frac{\eta_{\varepsilon}}{\delta n^{e f f}}\right)
$$

The strain-optic coefficient $\eta_{\varepsilon}$ accounts for the change in the refractive index of the fibre due to the applied strain. It was derived from the change in the optical indicatrix for the field polarized perpendicularly to the direction of the applied strain [25], $\eta_{\varepsilon}=-0.5 n^{3}\left(p_{12}-\mu\left(p_{12}+p_{11}\right)\right)$ where $\mathrm{p}_{11}=0.121$ and $\mathrm{p}_{12}=0.27$ are elements of the strain-optic tensor [25] and $\mu=0.17$ is the Poisson's coefficient for pure fused silica [26]. The calculated strain sensitivity was $-1.57 \mathrm{~nm} / \mathrm{m} \varepsilon$. 
The numerical solution was based on the change in the refractive index of silica of $\Delta n=\eta_{\varepsilon} \varepsilon=6 \cdot 10^{-4}$ caused by the maximal strain applied in the experiment of $2 \mathrm{~m} \varepsilon$. The beat length of the strained fibre was calculated in the range of $8 \mathrm{~nm}$ around the resonant wavelength. The new resonant wavelength was found by interpolation of the $\Lambda$ curves at the points determined by the new grating period $\Lambda=L(1+\varepsilon)$. From Fig. 5 it can be seen that both the contribution from the elongation of the grating (dashed arrow) and from the strain-optic effect (grey arrow) lead to a blue wavelength shift, the former having the major effect.

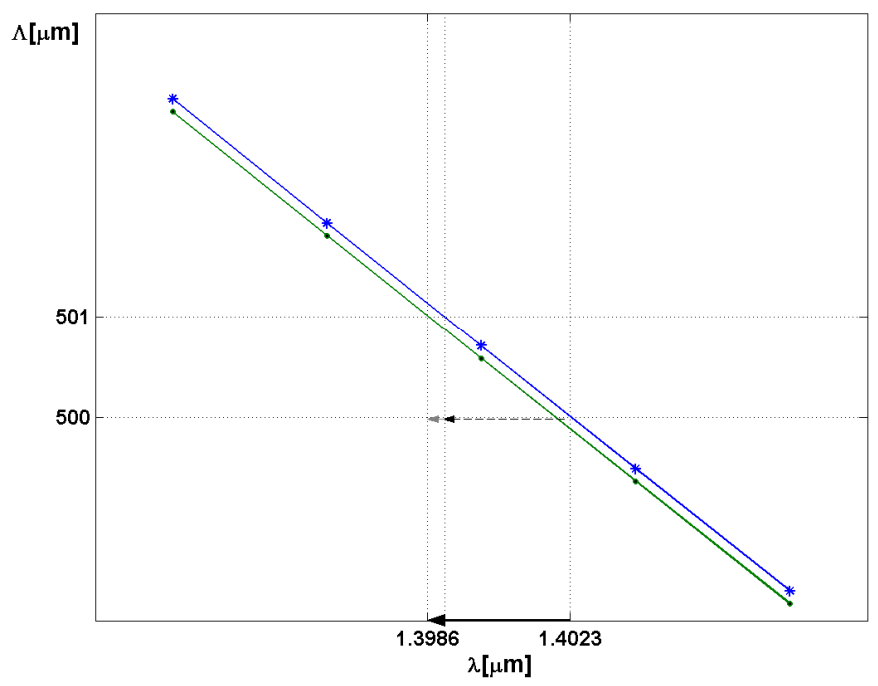

Figure 4. Numerical solution for the shift in the resonant wavelength of the LPG in ESM PCF due to the applied strain. * unstrained fibre, $\bullet$ - fibre under strain of $2 \mathrm{~m} \varepsilon$. Dashed arrow - wavelength shift due to the elongation of the fibre, grey arrow - wavelength shift due to the strain-optic effect, solid black arrow - overall wavelength shift.

The model was validated by comparing to the experimental results. The strain response of the LPG in ESM 1550-01 was linear with the sensitivity of $-2.08 \pm 0.05 \mathrm{~nm} / \mathrm{m \varepsilon}$, as shown in Fig. 5 .

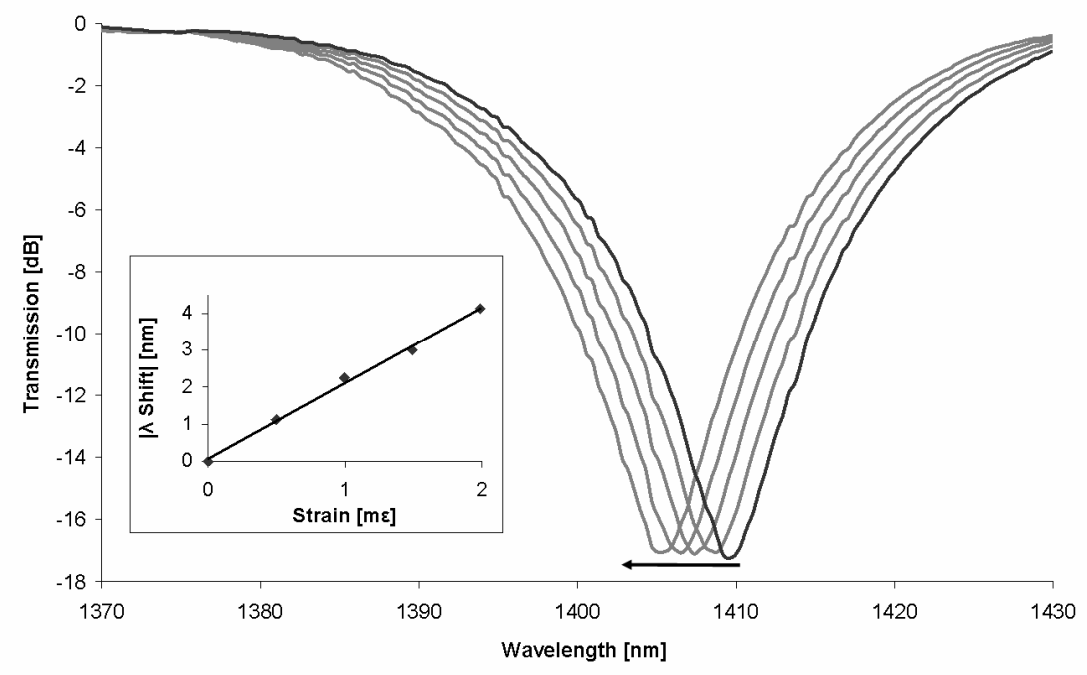

Figure 5. Measured sensitivity to strain of the attenuation band at $1403 \mathrm{~nm}$ of the $500 \mu \mathrm{m}$ LPG in ESM PCF.

The numerical result for the sensitivity of $-1.85 \mathrm{~nm} / \mathrm{m} \varepsilon$ differs from the measured value by $11 \%$, which is better than the error of the semi-analytical result of nearly $25 \%$. Apart from the experimental error of $2.4 \%$, the error of the model 
can be assigned to several neglected effects: inhomogeneous distribution of strain in the PCF caused by the presence of the air holes; dispersion of the strain-optic coefficients and average change in the refractive index in the grating. The simulation of the mechanical stresses in ESM PCF reveals that the axial strain in PCF reaches a maximum in the photonic crystal, Fig. 6. As the resonant mode is mainly concentrated in this part of the fibre (see Fig. 3), the variations in strain, calculated to be as large as $4 \%$, may be a source of error of the model. At the same time, the amplitude of the wavelength shift of only few nanometers makes the dispersion of the strain-optic coefficients negligible. The change in the refractive index of PCF exposed to the electric arc was not known at the time of the calculation hence the model was based on the unperturbed fibre. Later consideration, [27], showed that the index change is of the order of $10^{-4}$, which may lead to the error in the wavelength shift $\Delta \lambda=\frac{\partial \delta n^{\text {eff }}}{\partial n} \Delta n \Lambda$ of the order of $0.1 \mathrm{~nm}$ corresponding to the error in sensitivity of several percents. The model can be improved by taking into account the strain distribution from Fig. 6 and the estimate of the index change from [27]. This work is in progress.

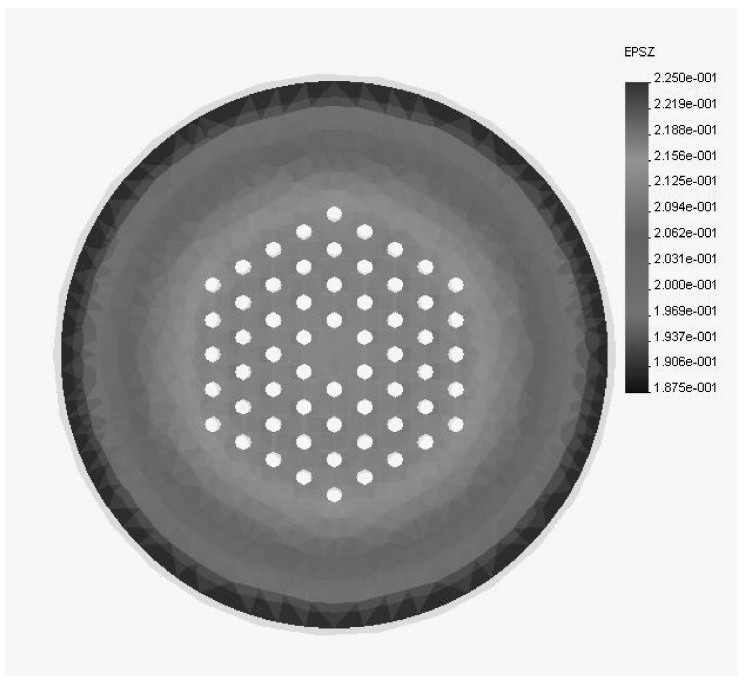

Figure 6. Distribution of the axial strain over the cross section of the ESM PCF. The figure is the courtesy of Tim EarthrowlGould (2004)

\section{LPG SENSITIVITY TO TEMPERATURE}

Similarly to strain, the expression for the LPG's sensitivity to temperature is a specific case of the generic expression given by Eq. (2)

$$
\frac{\partial \lambda}{\partial T}=\gamma \lambda\left(\alpha+\frac{\partial \delta n^{e f f}}{\partial n} \frac{\eta_{T}}{\delta n^{e f f}}\right)
$$

in which $\alpha=\partial \Lambda / \Lambda \partial T=5 \cdot 10^{-7}{ }^{\circ} \mathrm{C}^{-1}$ is the thermal expansion coefficient of silica and $\eta_{T}=\partial n / \partial T=7.8 \cdot 10^{-6}{ }^{\circ} \mathrm{C}^{-1}$ is the thermo-optic coefficient of silica [25]. For the range of temperatures applied in the experiment $\left(20^{\circ} \mathrm{C}-90^{\circ} \mathrm{C}\right)$ the maximal change in the refractive index is $5 \cdot 10^{-4}$, hence, under the assumption of linear grating response, the derivatives already calculated in the previous section could be used. The semi-analytical model gave sensitivity to temperature of $3.8 \mathrm{pm} /{ }^{\circ} \mathrm{C}$.

The numerical solution was obtained reusing the curves from Fig. 4 and following the steps outlined in the previous section. The change in the refractive index of $6 \cdot 10^{-4}$ corresponds to the temperature change of $-76.92^{\circ} \mathrm{C}$, which 
is just above the range used in our experiments. This caused only a small change in the grating period of $-19.23 \mathrm{~nm}$, Fig. 7 , corresponding to the sensitivity of $4.0 \mathrm{pm} /{ }^{\circ} \mathrm{C}$.

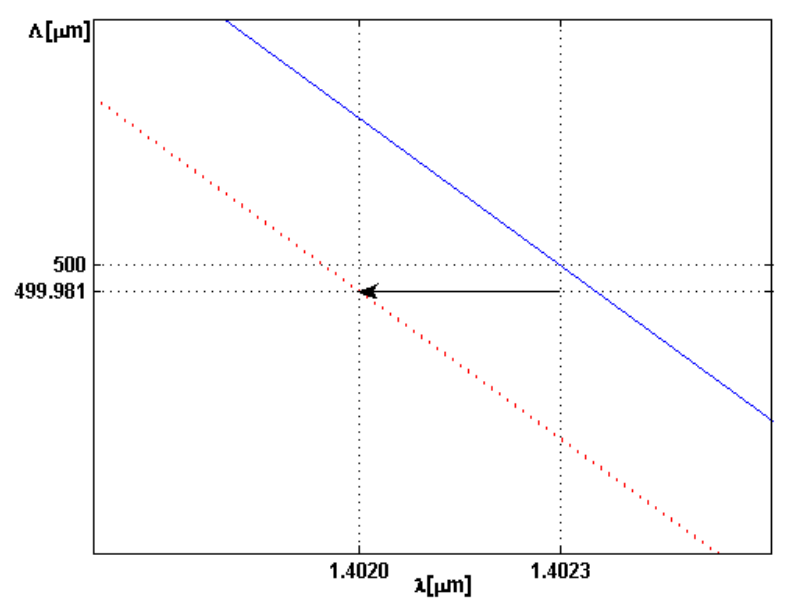

Figure 7. Numerical solution for the sensitivity of the LPG in ESM PCF to temperature when the grating is cooled by $77^{\circ} \mathrm{C}$.

Waveguide dispersion of the resonant mode is such that the thermo-optic effect and the thermal expansion of the fibre cause wavelength shifts of the opposite signs. Although the thermo-optic effect dominates the effect of linear expansion of the fibre, it is itself small because both core and cladding modes are guided mostly through the silica and their effective indices undergo almost the same change, hence the small value of the derivative $\partial \delta n^{\text {eff }} / \partial n=-1.17 \cdot 10^{-3}$. This explains the very weak sensitivity of the LPG to temperature and justifies the assumption used in the model that the volume expansion of the grating and thermal stresses can be neglected.

The sign and the order of magnitude of the calculated wavelength shift agree with the experimental result, but the calculated sensitivity overestimates the experimental value $2 \pm 1 \mathrm{pm} /{ }^{\circ} \mathrm{C}$. This disagreement may be due to the large error in the linear regression caused by scattering of the experimental data, but also due to the neglect of the average index change in the model of LPG and linearisation over the large temperature span. The error can be reduced by the improvements of the model described in the previous section.

\section{LPG SENSITIVITY TO EXTERNAL REFRACTIVE INDEX}

For refractive indices lower than the index of silica, the wavelength shift is a dominant grating response. It can be obtained from Eq. (3) by simply dropping the term that describes a change in the grating period:

$$
\frac{\partial \lambda}{\partial n_{e x t}}=\gamma \lambda \frac{1}{\delta n^{e f f}} \frac{\partial \delta n^{e f f}}{\partial n_{e x t}}
$$

Here the direct application of the linearisation around the resonant point is limited to indices far below the index of silica. On the other hand, the numerical model does not suffer from such a limitation and its implementation is straightforward as it is sufficient to set the refractive index of the ring simulating the surrounding material to the appropriate value. The calculated beat length shift for the LPG in ESM PCF versus the external refractive index is shown in Fig. 8a). Since change in the external index does not cause change in the grating period, the wavelength shift is simply obtained from the shift of the grating characteristic along the line $\Lambda=L=500 \mu \mathrm{m}$, Fig. 8b). The theoretical result 
correctly predicts the sign of the wavelength shift but overestimates the maximal measured value $0.8 \pm 0.1 \mathrm{~nm}$ [19] several times. We have not been able to explain this discrepancy.

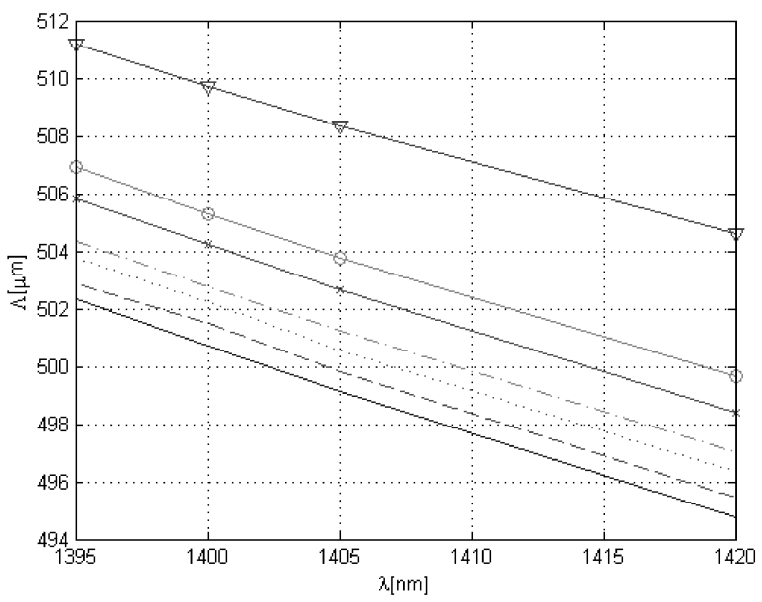

a)

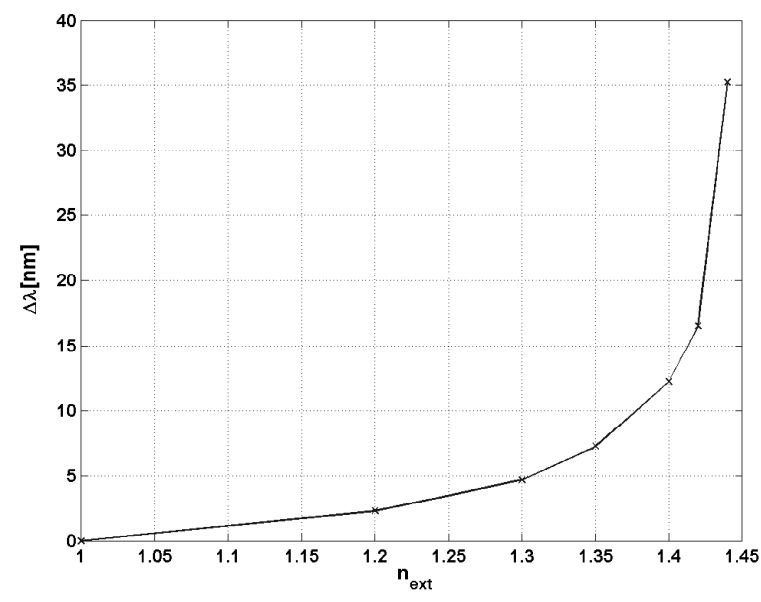

b)

Figure 8. a) Beat length of the resonant mode of the LPG in ESM PCF for different values of the external refractive index: 1.0 - solid line, 1.2 - dashed line, 1.3 - dotted line, 1.35 - dash-dot line, 1.4 - x, 1.42 - o, 1.44 - $\nabla$. b) Shift in the resonant wavelength obtained from a) versus external refractive index.

\section{CONCLUSION}

A numerical model of the sensitivity of long period grating fabricated in photonic crystal fibre to temperature, strain and refractive index has been proposed. Numerical results compared well to the measurements of the gratings fabricated by the electric arc in ESM PCF, with the exception of the index sensitivity, which is the subject of further study. In particular, the major property of these gratings which is the very low sensitivity to temperature has been explained, which paves the way to other similar solutions. The proposed numerical model was shown to be superior to the commonly used semi-analytical model. However, it allows for an improvement of accuracy by incorporating the estimated average induced index change and inhomogeneous distribution of axial strain.

\section{REFERENCES}

1. A. M. Vengsarkar, P. J. Lemaire, J. B. Judkins, V. Bhatia, T. Erdogan and J. E. Sipe, "Long-period gratings as bandrejection filters", J. Lightwave Technol. 14(1), 58-65 (1996)

2. C. Narayanan, H. M. Presby and A. M. Vengsarkar, "Band-rejection fibre filter using periodic core deformation", El. Lett. 33(4), 280-281 (1997)

3. S. W. James and R. P. Tatam, 'Optical fibre long-period grating sensors: characteristics and application', Measurement Science and Technology 14(5), 49 (2003)

4. T. Allsop, D. J. Webb, and I. Bennion, "A Comparison of the Sensing Characteristics of Long Period Gratings Written in Three Different Types of Fibre", Opt. Fib. Technol. 9, 210 (2003)

5. B. J. Eggleton, P. S. Westbrook, C. A. White, C. Kerbage, R. S. Windeler and G. L. Burdge, "Cladding-ModeResonances in Air-Silica Microstructure Optical Fibres”, J. Lightwave Technol 18(8), $1084-1099$ (2000) 
6. P. Russell, Science 299, 358-362 (2003)

7. H. Dobb, K. Kalli and D. J. Webb, "Temperature-insensitive long period grating sensors in photonic crystal fibre", El. Lett. 40, 657 (2004)

8. T. Allsop, D. J. Webb and I. Bennion, "A comparison of the spectral properties of high temperature annealed long period gratings inscribed by fs laser, UV and fusion-arc", Proceding of Photonics Europe (SPIE), 6193 (2006)

9. R. Kashyap, Fiber Bragg Gratings, Academic Press (1999)

10. A. Othonos, K. Kalli, Fiber Bragg Gratings, Artech House Inc., 192 (1999)

11. A. Ferrando, E. Silvestre, J. J. Miret, P. Andres and M. V. Andres, "Full-vector analysis of a realistic photonic crystal fiber", Opt. Lett. 24(5), 276-278 (1999)

12. W. Zhi, R. Guobin, L. Shuqin and J. Shuisheng. "Supercell lattice method for photonic crystal fibers", Opt. Express 11(9), 980-991 (2003)

13. T. P. White, B. T. Kuhlmey, R. C. McPhedran, D. Maystre, G. Renversez, C. M. de Sterke and L. C. Botten, "Multipole method for microstructured optical fibers. I. Formulation", J. Opt. Soc. Am. B 19(10), 2322-2330 (2002)

14. B. T. Kuhlmey, T. P. White, G. Renversez, D. Maystre, L. C. Botten, Lindsay, C. M. De Sterke and R. C. McPhedran, "Multipole method for microstructured optical fibers. II. Implementation and results", J. Opt. Soc. Am. B 19(10), 2331-2340 (2002)

15. Z. Zhu and T. G. Brown, Full-vectorial finite-difference analysis of microstructured optical fibres,", Opt. Express 10(17), 853-864 (2002)

16. H. P. Uranus and H. J. W. M. Hoekstra, "Modelling of microstructured waveguides using a finite-element-based vectorial mode solver with transparent boundary conditions", Opt. Express 12(12), 2795-2809 (2004)

17. F. Fogli, L. Saccomandi, P. Bassi, G. Bellanca, S. Trillo, "Full vectorial $\{$ BPM $\}$ modeling of index-guiding photonic crystal fibers and couplers", Opt. Express 10(1) 54-59 (2002)

18. P. Steinvurzel, E. D. Moore, E. C. Maegi, B. T. Kuhmley and B. J. Eggleton, "Long peirod grating resonances in photonic bandgap fibre", Opt. Express 14(7) 3007- 3014 (2006)

19. H. Dobb, K. Kalli, D. J. Webb, 'Measured sensitivity of arc-induced long-period grating sensors in photonic crystal fibre', Opt. Comm. 260(1), 184 (2006)

20. Comsol Inc., http://www.comsol.com

21. P. R. McIsaac, IEEE Trans. Microwave Theory Tech. MTT-23, 421 (1975)

22. T. Erdogan, J. Opt. Soc. Am. A 14(8), 1760-1773 (1997)

23. T. W. MacDougall, S.Pilevar, C. W. Haggans and M. A. Jackson, Phot. Technol. Lett. 10(10), 1449 (1998)

24. X. Shu, L. Zhang and I. Bennion, J. Lightwave Technol. 20(2), 255-266 (2002)

25. G. B. Hocker, Applied Optics 18( 9) 1445 (1979)

26. R. Gunter, Modern Optics, John Wiley and Sons, Inc. (1990)

27. J. S. Petrovic, D. J. Webb, H. Dobb, V. Mezentsev, K. Kalli and I. Bennion, "Nondestructive Index Profiling of Long Period Gratings in Photonic Crystal Fibres", Opt Quant El. 38(9-11), 913-920 (2006) 